\title{
High-Intensity Interval Exercise: Methodological Considerations for Behavior Promotion From an Affective Perspective
}

\author{
Allyson G. Box* and Steven J. Petruzzello \\ Department of Kinesiology and Community Health, University of Illinois Urbana-Champaign, Champaign, IL, United States
}

Keywords: HIIT, physical activity, valence, pleasure, HIFT, affect

High-intensity interval exercise and high-intensity interval training (HIIT), an exercise approach alternating short bouts of vigorous exercise with less intense recovery or rest periods, has been rated as a leading fitness trend, ranked between $\# 1$ and \#3 in the annual survey of "Top 20 Worldwide Fitness Trends" since 2014, with no sign of weakening "popularity" (Thompson, 2019). This increased popularity in HIIT programs has been mirrored by a subsequent uptick in related research. In such investigations, HIIT appears to deliver important physiological benefits (much like those observed by any regular exercise behavior; Kilpatrick et al., 2014; Jelleyman et al., 2015). However, little is known about why high-intensity interval programs have gained such popularity within the fitness industry (i.e., increasing number of high-intensity interval type franchises such

\section{OPEN ACCESS}

Edited by:

David M. Williams,

Brown University, United States

Reviewed by:

Lauren Bohlen,

Brown University, United States

${ }^{*}$ Correspondence:

Allyson G. Box

agbox2@illinois.edu

Specialty section:

This article was submitted to

Movement Science and Sport

Psychology,

a section of the journal

Frontiers in Psychology

Received: 19 May 2020 Accepted: 06 January 2021 Published: 28 January 2021

Citation:

Box AG and Petruzzello SJ (2021)

High-Intensity Interval Exercise: Methodological Considerations for Behavior Promotion From an Affective

Perspective.

Front. Psychol. 12:563785

doi: 10.3389/fpsyg.2021.563785 as F45 ${ }^{\circledR}$ Training, Orangetheory Fitness ${ }^{\circledR}$, Crossfit ${ }^{\circledR}$ Training, Bootcamps, and so on), let alone, and perhaps most importantly, whether such a regimen encourages prolonged exercise ${ }^{1}$ behavior (i.e., adherence). Based on previous work (e.g., Williams et al., 2008) with continuous exercise, the affective experience of such HIIT-type programs may be a particularly important reason why it is popular. Thus, we will address some methodological concerns pertaining to HIIT research, specifically related to the study of affective states. We will also propose potential solutions for investigating such psychological phenomena associated with this popular exercise regimen.

\section{DISENTANGLING HIIT TERMINOLOGY}

HIIT, by definition, utilizes planned intensity and work to rest/recovery ratios in unlimited variations (see Laursen and Buchheit, 2019, Figure $\mathbf{1}$ for detail on intensity and design variability). That is, a ratio defined as 1:1 (keeping in mind that numerous ratios can be delineated) may differ in duration (e.g., 30-s work: 30-s rest/recovery; 2-min work: 2-min rest/recovery) and exercise type (e.g., running; cycling; also consider that body weight and resistance circuits often include multiple movements within a single HIIT session). These types of protocols are almost exclusively done in research. A closely related regimen, referred to as HIFT, is defined as a "training style that incorporates a variety of functional movements, performed at high-intensity (relative to an individual's ability), to improve parameters of general physical fitness and performance" (Feito et al., 2018, p. 2).

HIFT is distinct from HIIT in at least two important ways: (a) rest/recovery and (b) intensity. While rest/recovery in research-based HIIT is planned and synchronous, HIFT provides greater

\footnotetext{
${ }^{1}$ While the field often uses the term "exercise" behavior/promotion, this should be interpreted as physical activity behavior and promotion, not simply exercise. The goal is to increase the total amount of physical activity, which may be accumulated via any bodily movement. Exercise, by definition, is planned, structured, repetitive physical activity that is often performed to improve one or more aspects of health (e.g., physical, mental, and social) and fitness (e.g., cardiorespiratory endurance, muscular strength and endurance, flexibility; American College of Sports Medicine et al., 2018).
} 
autonomy in both rest and recovery. That is, the individual makes the determination of when, and for how long, to rest/recover. Another consideration is whether "high-intensity" is an adequate term to express the intensity performed. Due to the flexibility in high-intensity interval programming, laboratory studies impose a range of intensities from just within "vigorous" intensity all the way to "supra-maximal" (Laursen and Buchheit, 2019). However, the fitness communities (e.g., F45 ${ }^{\circledR}$ Training, Orangetheory Fitness ${ }^{\circledR}$, Crossfit ${ }^{\circledR}$ Training, Bootcamps, and so on) applying "high-intensity interval-type" programming encourage the individual to "exercise as hard as you can," which may or may not equate to a physiological index of high intensity (see American College of Sports Medicine et al., 2018, p. 146). It is possible the majority of individuals ${ }^{2}$ engaging in leisure- or health-related "interval-type" exercise are most often engaging in self-selected, perceived high-intensity (i.e., completely autonomous) interval-type exercise. Thus, intensity itself is perceptual in nature and varies during this type of exercise session. As a result, the individual is likely modifying their workto-recovery ratio, and subsequently their intensity, based on how they are feeling (i.e., their affective experience). It is entirely possible these "interval-type" programs remain a popular form of exercise due to the autonomy in both level of exercise-intensity and rest/recovery, resulting in an affective quality (e.g., less unpleasant) that enhances affective associations toward exercise.

\section{MAXIMIZING QUALITY OF RESEARCH ON AFFECTIVE RESPONSES}

Psychological Hedonism is, stated simply, the idea that human behavior is a result of an innate pursuit of pleasure and avoidance of displeasure (Young, 1952; Rozin, 1999). Indeed, accumulating evidence suggests in-task (i.e., during exercise) affective states (i.e., pleasure vs. displeasure) are associated with an increased likelihood of continued exercise behavior, even up to 6- and 12-months later (Williams et al., 2008; see also Rhodes and Kates, 2015 for a review). While we will not go as far to say hedonics is the only, or even primary, concept to be considered in human behavior, we do implore that affective states (also considered feeling states $^{3}$ ) should at least be considered. As Antonio Damasio (1994) eloquently wrote -

"Knowing about the relevance of feelings in the process of reason does not suggest that reason is less important than feelings, that it

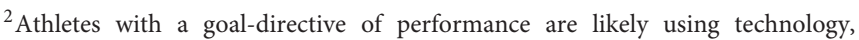
providing a physiological index, to assure they are adequately exercising within a high-intensity range.

${ }^{3}$ Feeling states are more commonly referred to as affective states within the literature and consist of three similar, but distinct, components: core affect, emotion, and mood. Core affect is the most basic, consciously accessible feeling state and is considered to reflect both affective valence (i.e., pleasure vs. displeasure) and activation (i.e., reflecting the psychological state of wakefulness). Emotion is an "intense" fluctuation in core affect following an internal or external event, real or imagined, that dissipates in a relatively quick (i.e., seconds) timeframe. Moods are longer in duration (i.e., hours or days), less intense (i.e., degree of fluctuation in core affect), and are distinguished by their onset ambiguity (see Ekkekakis, 2013, Nowlis and Nowlis, 1956, and Russell, 2003 for further detail on affective states).
}

should take a backseat to them or that it should be less cultivated. On the contrary, taking stock of the pervasive role of feelings may give us a chance of enhancing their positive effects and reducing their potential harm (p. 246)."

With so much yet to learn about feeling states in the context of exercise behavior, the relationship between exercise intensity and affective valence (i.e., pleasure vs. displeasure) has been well-established. Evidence has repeatedly demonstrated the ventilatory threshold (VT) as the biological marker of most importance for influencing affective states, that is, fluctuations in one's affective state reliably occur depending on the exercise intensity relative to the VT. Intensities below the VT elicit very little fluctuation in affective state and result in primarily homogenous in-task pleasure, while exercise intensities above the VT elicit intense fluctuation in affective state and result in homogenous in-task displeasure. More fascinating is the effect of exercise intensity at, or proximal to, the VT. It is within this intensity range where heterogeneity of affective responses exists, with such heterogeneity being attributed to fitness, personality, and other individual differences (Acevedo et al., 2003; Ekkekakis et al., 2005; Box and Petruzzello, 2020).

While yet to be empirically tested, it is likely individuals who regularly engage in (self-selected, perceived) high-intensity interval type exercise (i.e., not in laboratory-based studies) are choosing to perform at an intensity at or proximal to their VT when prompted to "exercise as hard as you can." Again, it is likely the autonomy in exercise-intensity and rest/recovery (adjusting both exercise-intensity and rest/recovery when it feels necessary) allows for more effective affective rebounds. Further, these affective rebounds, occurring immediately following exercise cessation within each of the rest/recovery intervals and at the end of the overall exercise session (see Figure 1, revised from Box et al., 2020 to demonstrate interval-exercise affective rebounds), likely result in a different affective response than if intensity or rest/recovery were imposed at preplanned intervals. Thus, experiencing decreases in pleasure (or increases in displeasure) within successive imposed highintensity work intervals likely is experienced very differently than when the work-to-recovery intervals are self-selected (i.e., autonomous). Differences in affective responses have been observed with a slight preference toward self-selected vs. imposed continuous exercise (Oliveira et al., 2015), but this has yet to be empirically demonstrated during intervaltype exercise.

\section{CONSIDERATIONS FOR EXPERIMENTAL DESIGN}

High-intensity interval exercise is not, nor will any exercise mode ever be, the only solution for increasing exercise behavior. This is not to say that high-intensity exercise research is frivolous; rather quite the opposite. An attempt should be made to best understand how exercise of any variety influences an individual, physiologically and psychologically, and how the individual chooses and adheres to different modes of activity. We are urging the utmost 


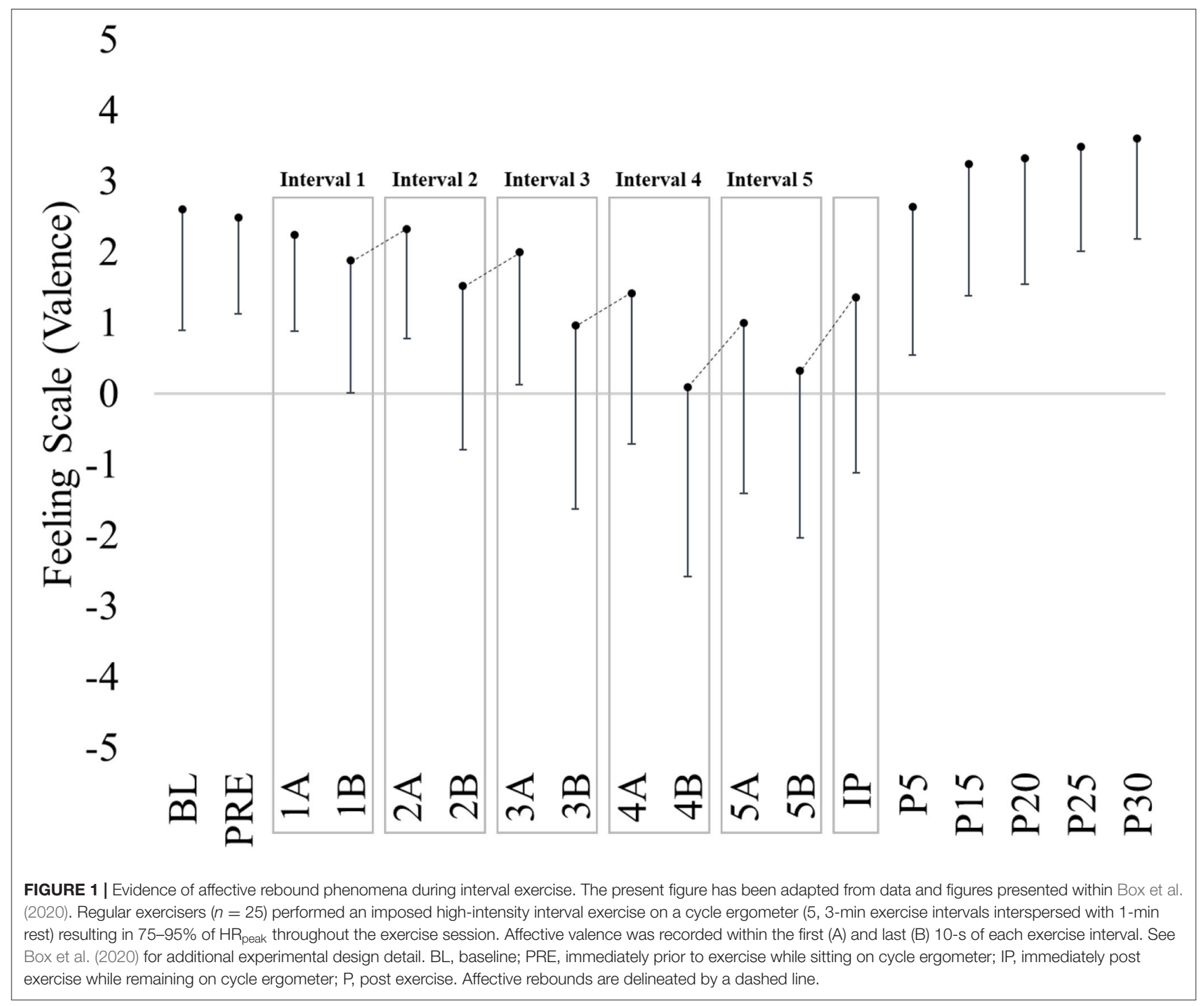

caution in designing and implementing research to examine such high-intensity, interval type exercise by thoughtfully considering the question at hand, deliberately designing a protocol that unequivocally tests these questions, and transparently interpreting the findings so as not to obscure the answers within.

We strongly encourage the following considerations when examining high-intensity interval-type exercise with an eye toward exercise promotion. We believe the inclusion and transparency of these variables will aid in comparing and interpreting findings across the "interval-type" literature.

We suggest that investigators:

a) Appropriately acquire and report a physiological index of intensity (e.g., \%HR $\left.R_{\text {max }}, \% V O_{2 p e a k}\right)$ and/or total accumulated work. Given the demonstrated importance of intensity on affective responses, this is a crucial methodological step. The decision of which physiological intensity index is dependent on the research question and experimental limitations, but whenever possible there should not be a sole reliance on perceived exertion.

b) Attempt to acquire and quantify VT alongside in-task affect. The importance of this marker stems from the well-established evidence that affective responses are most aligned with the VT rather than a percentage of maximum heart rate or $\mathrm{VO}_{2 \text { peak }}$. This is important because at the same physiological index of intensity (e.g., $\% \mathrm{VO}_{2 \text { peak }}$ ), one person could be well above their VT and experience the exercise as unpleasant while another person is well under their VT and experiences the exercise as pleasant. Thus, it is more informative to have a participant's in-task affect displayed and interpreted in relation to their VT. This also allows for easier comparisons across studies implementing various interval designs and exercise movements. 
c) Record affective states during both work and rest/recovery periods along with pre- and post-exercise. The time-ofassessment should be identical between any compared conditions (especially interval vs. continuous exercise), meticulously designing the assessments to occur during exercise for both conditions. The timing should be such that affective states are acquired at least immediately prior to the exercise session (within 1-min), during exercise and rest/recovery intervals, and immediately post (within 1-min) exercise session. At minimum, affective states should be assessed within the first and last 15-s of each exercise interval and halfway through rest/recovery. Waiting to assess affect until the work bout has just ended will not capture the same affective dynamic as the affective rebound is likely already taking place at that time. Participant reactivity to repeated assessments is also possible when acquiring affective states several times within a short time period, thus forethought is needed to determine how often it is necessary to assess affective states. See Ekkekakis (2013) for suggestions on affective state questionnaires.

d) Manipulate only one exercise variable (i.e., intensity, duration, or mode) while standardizing all other variables between testing conditions. It is necessary, until evidence has been established, to manipulate only one exercise variable to determine whether affective states are a consequence of intensity, duration, or mode. Too often in the highintensity interval-type vs. moderate-intensity continuous exercise literature all three variables are manipulated, resulting in muddled interpretations. This will likely require multiple experiments in order to confidently provide inferences to a single question, but careful planning that allows for both replication and extension of findings should provide more confidence in the findings.

e) Preemptively control, recognize, record, and report possible extraneous variables. Affective states, by definition, fluctuate moment-to-moment and many variables could unduly

\section{REFERENCES}

Acevedo, E. O., Kraemer, R. R., Haltom, R. W., and Tryniecki, J. L. (2003). Perceptual responses proximal to the onset of blood lactate accumulation. $J$. Sports Med. Phys. Fit. 43, 267-273.

American College of Sports Medicine, Riebe, D., Ehrman, J. K., Liguori, G., and Magal, M. (2018). ACSM's Guidelines for Exercise Testing and Prescription. Philadelphia, PA: Wolters Kluwer.

Box, A. G., Feito, Y., Zenko, Z., and Petruzzello, S. J. (2020). The affective interval: an investigation of the peaks and valleys during high-and moderateintensity interval exercise in regular exercisers. Psychol. Sport Exerc. 49:101686. doi: 10.1016/j.psychsport.2020.101686

Box, A. G., and Petruzzello, S. J. (2020). Why do they do it? Differences in high-intensity exercise-affect between those with higher and lower intensity preference and tolerance. Psychol. Sport Exerc. 47:101521. doi: 10.1016/j.psychsport.2019.04.011

Damasio, A. R. (1994). Descartes' Error: Emotion, Reason, and the Human Brain. New York, NY: Putnam.

Ekkekakis, P. (2013). The Measurement of Affect, Mood, and Emotion: A Guide for Health-Behavioral Research. New York, NY: Cambridge University Press. doi: 10.1017/СBO9780511820724 influence an individual's affective state data. Consider the lab environment (e.g., decorative pictures, number of research staff, music, unintended conversation, etc.), the researcher's appearance and tone (e.g., white coat affect, provocative clothing, excited vs. bored tone, etc.), the participant's personal items (e.g., cell phone, smart watch, etc.), and so on to eliminate as many potentially confounding sources as possible.

\section{CONCLUSION}

HIIT programs and the numerous variations that have evolved are popular and seem destined to be part of the exercise landscape. Our position in this paper is that we, as exercise behavior researchers, need to exert much greater care in the way that we study these high-intensity interval options. We have outlined what we think are the most critical issues in the design and execution of research, particularly as related to understanding the affective dynamics of such exercise. Given that affect has been shown to be intimately linked with exercise intensity and that affect experienced during exercise is consistently predictive of adherence, careful examination of these affective dynamics in high-intensity interval exercise is crucial. The bottom line is that, as popular as some forms of activity might be, if people do not experience them in such a way that engenders long-term adherence, which is likely if the activity is experienced as unpleasant, it really does not matter what physiological benefits might be gained. We have a lot of work to do to achieve this level of understanding, particularly with high-intensity interval exercise.

\section{AUTHOR CONTRIBUTIONS}

$A B$ was responsible for initial drafting, while both $A B$ and SP equally contributed to the content and draft finalizing. Both authors contributed to the article and approved the submitted version.

Ekkekakis, P., Hall, E. E., and Petruzzello, S. J. (2005). Variation and homogeneity in affective responses to physical activity of varying intensities: an alternative perspective on dose-response based on evolutionary considerations. J. Sports Sci. 23, 477-500. doi: 10.1080/02640410400021492

Feito, Y., Heinrich, K. M., Butcher, S. J., and Carlos Poston, W. S. (2018). Highintensity functional training (HIFT): definition and research implications for improved fitness. Sports 6:76. doi: 10.3390/sports6030076

Jelleyman, C., Yates, T., O’Donovan, G., Gray, L. J., King, J. A., Khunti, K., et al. (2015). The effects of high-intensity interval training on glucose regulation and insulin resistance: a meta-analysis. Obes. Rev. 16, 942-961. doi: $10.1111 /$ obr.12317

Kilpatrick, M. W., Jung, M. E., and Little, J. P. (2014). High-intensity interval training: a review of physiological and psychological responses. ACSMs Health Fit. J. 18, 11-16. doi: 10.1249/FIT.0000000000000067

Laursen, P., and Buchheit, M. (2019). Science and application of high-intensity interval training. Hum. Kinet. doi: 10.5040/9781492595830

Nowlis, V., and Nowlis, H. H. (1956). The description and analysis of mood. Ann. N Y Acad. Sci. 65, 345-355. doi: 10.1111/j.1749-6632.1956.tb49644.x

Oliveira, B., Deslandes, A., and Santos, T. (2015). Differences in exercise intensity seems to influence the affective responses in self-selected and imposed exercise: a meta-analysis. Front. Psychol. 6:1105. doi: 10.3389/fpsyg.2015.01105 
Rhodes, R. E., and Kates, A. (2015). Can the affective response to exercise predict future motives and physical activity behavior? A systematic review of published evidence. Ann. Behav. Med. 49, 715-731. doi: 10.1007/s12160-015-9704-5

Rozin, P. (1999). "Preadaptation and the puzzles and properties of pleasure," in Well-being: The Foundations of Hedonic Psychology, eds D. Kahneman, E. Diener, and N. Schwarz (New York, NY: Russell Sage), 109-133.

Russell, J. A. (2003). Core affect and the psychological construction of emotion. Psychol. Rev. 110, 145-172. doi: 10.1037/0033-295X.110.1.145

Thompson, W. R. (2019). Worldwide survey of fitness trends for 2020. ACSMs Health Fit. J. 22, 10-17. doi: 10.1249/FIT.0000000000000438

Williams, D. M., Dunsiger, S., Ciccolo, J. T., Lewis, B. A., Albrecht, A. E., and Marcus, B. H. (2008). Acute affective response to a moderate-intensity exercise stimulus predicts physical activity participation 6 and 12 months later. Psychol. Sport Exerc. 9, 231-245. doi: 10.1016/j.psychsport.2007.04.002
Young, P. T. (1952). The role of hedonic processes in the organization of behavior. Psychol. Rev. 59, 249-262. doi: 10.1037/h0057176

Conflict of Interest: The authors declare that the research was conducted in the absence of any commercial or financial relationships that could be construed as a potential conflict of interest.

Copyright $\odot 2021$ Box and Petruzzello. This is an open-access article distributed under the terms of the Creative Commons Attribution License (CC BY). The use, distribution or reproduction in other forums is permitted, provided the original author(s) and the copyright owner(s) are credited and that the original publication in this journal is cited, in accordance with accepted academic practice. No use, distribution or reproduction is permitted which does not comply with these terms. 